\title{
ASSOCIATION BETWEEN EXPOSURE TO FAMILY ENVIRONMENTAL TOBACCO SMOKE AND OBSTRUCTIVE SLEEP APNEA HYPOPNEA SYNDROME
}

\author{
HUANG, Q. ${ }^{1}-$ ALI, S. ${ }^{2}-$ ZHANG, J. ${ }^{1 *}$ \\ ${ }^{I}$ Department of Respiratory Medicine, Beijing Tian Tan Hospital, Capital Medical University, \\ Beijing, China \\ ${ }^{2}$ Department of Information Science, University of Education, Lahore, Pakistan \\ *Corresponding author \\ e-mail:ttyy_zj@163.com \\ (Received $23^{\text {rd }}$ May 2019; accepted $4^{\text {th }}$ Jul 2019)
}

\begin{abstract}
The objective of this study was to observe the relationship between levels of family environmental tobacco smoke (ETS) and obstructive sleep apnea hypopnea syndrome (OSAHS). Clinical datr AHI, lower oxygen saturation, longer apnea time, and lower ventilation time $(p<0.05)$. There was a correlation between EST and OSAHS. Compared with non-exposa of 209 patients who visited the respiratory department were retroactively collected. The data entailed gender, age, body mass index (BMI), family environmental smoke exposure, medical history, Epworth sleepiness scale (ESS) score, and polysomnography (PSG) indicators. Two groups of patients were studied: ETS-exposed group and non-ETS-exposed group. Using the apnea hypopnea index (AHI), OSAHS patients were classified as mild $(5 \leq \mathrm{AHI} \leq 15)$, moderate $(15<\mathrm{AHI} \leq 30)$, and severe (AHI > 30). There were 148 cases in the OASHS group and 61 cases in the normal group. 56.66\% of the OASHA group were ETS-exposed patients, which was significantly higher than that the normal group ETSexposure of $31.57 \%$. Compared to the OSAHS patients unexposed to ETS, the OSAHS patients with EST exposure had a greater degree of lethargy, greateed, the EST-exposed group was more susceptible to OSAHS, and the longer the time, the more severe the condition.
\end{abstract}

Keywords: obstructive sleep apnea hypopnea syndrome, health effects, passive smoking, sleep apnea hypopnea index, ESS questionnaire

\section{Introduction}

Environmental tobacco smoke (EST) exposure has become a worldwide health problem. Currently, there are 1.3 billion smokers worldwide, and a high number of people are exposed to family environmental tobacco smoke (Rando et al. 999; Aviado et al., 1996). Studies have shown that EST impairs the stability of the internal environment and affects normal physiological processes, leading to various chronic diseases. Obstructive sleep apnea hypopnea syndrome (OSAHS) accounts for most of sleep apnea hypopnea syndrome cases, a frequently-occurring disease (Muggli, 2004). In China, OSAHS affects about $4 \%$ of adults. The syndrome is characterized by snoring and repeated apnea resulting in sleep fragmentation, morning discomfort, dry mouth, daytime sleepiness, fatigue, dizziness, headache, decreased memory, and inattentiveness. These problems result in reduced work efficiency, increased risk of industrial accidents and traffic accidents, as well as sudden death during sleep (Davidson and Reed, 2018; Theweny, 2015). With improvement in living standards, ageing and incidence of obesity have increased significantly, and the incidence of OSAHS is increasing year by year, thereby posing an important public health problem. Since OSAHS and ETS often occur simultaneously, they may have common oxidative stress and inflammatory pathways, leading to metabolic disorders (Barnes et al., 2006; Elliott et al., 2007; Jenkins and Counts, 1999; Steenland, 1999; Thun et al., 1999). At 
present, local and foreign scholars have studied the etiology and pathogenesis of OSAHS. Studies have suggested that ETS is one of the risk factors for OSAHS, but there are few studies on the relationship between ETS and OSAHS.

In the present study, the relationship between EST and OSAHS was studied by investigating the association between ETS exposure and the severity of OSAHS, so as to provide a theoretical basis for effective prevention and treatment of OSAHS.

\section{Materials and methods}

\section{Materials}

Setting and samples

The clinical data of 209 patients who visited the Department of Respiratory Medicine were retrospectively collected. The patients were divided into ETS-exposed group and nonETS-exposed group. The ETS-exposed group was sub-divided into mid-ETS (tobacco smoke in the house but not in the same room as patient), and heavy-ETS (tobacco smoke in the same room as patient). Using the latest American Society of Sleep Medicine Standards (AASM) and AHI, patients with OSAHS were classified as mild $(5 \leq \mathrm{AHI} \leq 15)$, moderate $(15<\mathrm{AHI} \leq 30)$, and severe (AHI > 30). The OSAHS classification is shown in Table 1.

Table 1. Grading of OSAHS

\begin{tabular}{c|c|c}
\hline Classification & AHI (time/hour) & LSaO $_{\mathbf{2}}(\boldsymbol{\%})$ \\
\hline Mild & $5-15$ & $85-90$ \\
Moderate & $>15-30$ & $80-<85$ \\
Severe & $>30$ & $<80$ \\
\hline
\end{tabular}

\section{Inclusion criteria}

Patients older than 18 years, patients who snored while sleeping at night and in daytime, and those with complete clinical data (including BMI, ESS score and PSG monitoring, etc.) were included in the study.

\section{Exclusion criteria}

Patients with loss of polysomnography, maxillofacial deformity, severe pneumonia, asthma, pulmonary interstitial disease, and chronic obstructive pulmonary disease were excluded. Moreover, those with severe heart failure, liver failure, renal failure, and neuromuscular diseases were excluded. Patients with central and mixed sleep apnea, and those on prolonged use of sleeping and sedative drugs were considered ineligible.

\section{Methods}

\section{Polysomnography}

Polysomnography (PSG) is the most important test for diagnosing OSAHS. Through continuous monitoring of night-time breathing, arterial oxygen saturation, electroencephalogram, electrocardiogram, heart rate and other indicators, you can know whether the snorer has apnea, the number of pauses, the time of suspension, the minimum arterial oxygen value at the time of suspension, and The extent of the impact on physical 
health is an internationally recognized gold standard for the diagnosis of OSAHS. The polysomnography is the most commonly used sleep monitoring method and is the internationally recognized gold standard for diagnosing OSAHS. According to the results of polysomnography, not only can the diagnosis of OSAHS be made, but also the severity can be judged, and it is convenient to formulate clinical treatment plans and quantitatively evaluate the operation or other treatment effects. Polysomnography is used to measure various physiological signals during sleep. The parameters of the measurement include: (1) Breathing: The airflow monitor can find the length and frequency of sleep apnea syndrome. Stopping breathing for more than $10 \mathrm{~s}$ is called a pause. Insufficient breathing usually means that the respiratory airflow drops by more than 50\%. (2) Oxygen saturation: A decrease in blood oxygen saturation is a key manifestation of OSAHS patients. (3) Muscle movement: Monitoring of chest movements can help diagnose whether the pause is central or obstructive. Other types of sleep disorders can be manifested as leg movements, jaw closures, and other characteristic sports. (4) Brain waves and eye movements: Sleep can be divided into different stages according to brain waves and eye movements, namely fast phase and slow phase (deep and light sleep). (5) Electrocardiogram (ECG): Some patients with OSAHS may have abnormal heart rhythms. (6) Position of the body: OSAHS is most likely to occur in the supine position, when the base of the tongue falls and it is easy to block the upper respiratory tract. Two of these assessment methods are often cited to summarize sleep monitoring outcomes: (1) Calculate the mean of the hourly breathing disorder by calculating the average of the sum of night-time sleep apnea syndrome and hypopneas, ie, breathing Pause the hypogastric index Apnea Hypopnea Index (AHI). Adult AHI greater than or equal to 5 can be defined as OSAHS. (2) The lowest oxygen saturation in sleep apnea syndrome, although there is no specific indication of sleep apnea syndrome, but less than $85 \%$ can determine sleep apnea syndrome. The PSG monitor with US Embla NN7000 (Jain, 2018) multi-lead sleep monitor was used to monitor the subjects for polysomnography $\geq 7 \mathrm{~h}$ overnight. At the same time, the subjects were advised to avoid drinking alcohol, coffee, tea, taking sleeping pills, and sedating drugs. The monitored parameters were EEG, ocular electricity, mandibular muscle power, nose and mouth airflow, chest and abdomen exercise, ECG, eye movement, body position, snoring, and fingertip oxygen saturation. The results were analyzed with computer software, and were strictly followed by professional physicians in accordance with the American Sleep Medicine Society. The sleep monitoring indicators selected for reflecting the objective severity of the patient's disease were AHI, minimum oxygen saturation, mean oxygen saturation, longest apnea time, and longest low ventilation time.

\section{Tests and questionnaires}

The clinical data of all selected subjects were collected. These were gender, age, height, weight, past medical history (hypertension, diabetes, cardiovascular and cerebrovascular diseases). The ESS score, AHI, minimum oxygen saturation, mean oxygen saturation, longest apnea time, and longest low ventilation time were recorded. The ESS shown in Table 2 was used for the subjects. The ESS was assessed by a professional under the guidance of a questionnaire containing 8 questions, each with 0-3 points and 4 levels. The maximum score was 24 points. Determination of lethargy ESS score < 5 is normal, 5-9 is mild sleepiness, 10-15 is moderate sleepiness, 16-24 is severe sleepiness. The higher the score, the more severe the daytime sleepiness, and scores greater than 10 points were considered to be excessive daytime sleepiness. 
Table 2. The Epworth sleepiness scale (ESS) score chart

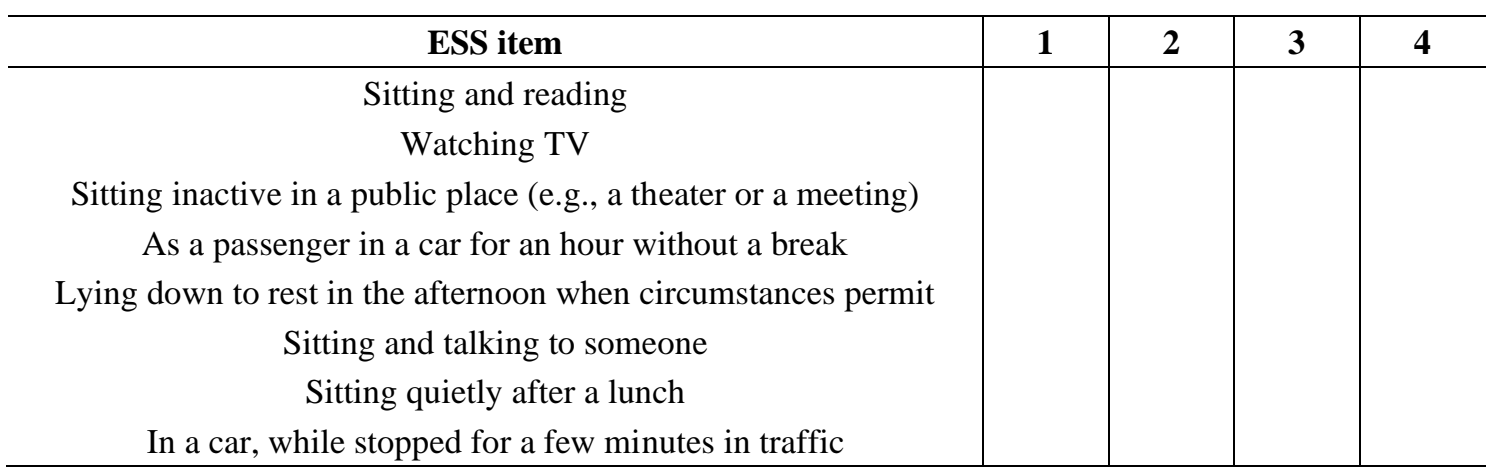

\section{Statistical analysis}

Normal distribution data are presented as mean \pm standard deviation $(\overline{\mathrm{x}} \pm \mathrm{s})$. Statistical differences were analyzed with $\chi^{2}$ test and $t$-test. All statistical analyses were performed using SPSS 23. Differences were assumed statistically significant at $p<0.05$.

\section{Results}

\section{Characteristics of participants}

A total of 209 patients were included in the study, out of which 148 patients had OSAHS, comprising 111 males and 37 females. The remaining patients comprised 37 males and 24 females. The population of OSAHS patients with EST exposure formed $56.66 \%$, which was significantly higher than that in the normal group (31.57\%), and the OSAHS group had significantly higher BMI $(p<0.05)$. There were no significant differences in age, and in male and female distribution $(p>0.05$; Table 3$)$.

Table 3. Characteristics of the OSAHS group and the normal group

\begin{tabular}{c|c|c|c|c}
\hline Parameter & OSAHS group & Normal group & Test value & $\boldsymbol{p}$ \\
\hline Gender (male/female) & $111 / 37$ & $34 / 24$ & 2.191 & 0.162 \\
Age (years) & $48.17 \pm 12.41$ & $46.71 \pm 11.56$ & -0.659 & 0.427 \\
BMI $\left(\mathrm{kg} / \mathrm{m}^{2}\right)$ & $29.58 \pm 4.28$ & $25.89 \pm 1.37$ & 3.179 & $0.001^{* *}$ \\
EST-exposed group (\%) & $87(58.89)$ & $19(30.56)$ & 5.728 & $0.009^{* *}$ \\
\hline${ }^{*} p<0.05,{ }^{* *} p<0.01$ &
\end{tabular}

\section{Comparative analysis of OSAHS with EST exposure and the non-ETS-exposed group}

There were 87 cases in OSAHS group with EST exposure consisting of 77 males and 10 females. In the non-ETS-exposed OSAHS group, there were 61 subjects made up of 34 males and 27 females. Compared with OSAHS group without exposure to ETS, OSAHS group with EST exposure differed significantly in gender composition $(p=0.000)$, but there were no significant differences in age, BMI and past medical history between the two groups $(p>0.05)$. These results are shown in Table 4 . 
Table 4. Clinical data of OSAHS group exposed to EST, and OSAHS group unexposed to ETS

\begin{tabular}{c|c|c|c|c}
\hline Parameter & $\begin{array}{c}\text { OSAHS group with } \\
\text { EST exposure }\end{array}$ & $\begin{array}{c}\text { OSAHS group } \\
\text { within no-exposed }\end{array}$ & Test value & $\boldsymbol{p}$ \\
\hline Gender (male/female) & $77 / 10$ & $34 / 27$ & 11.787 & $0.000^{* *}$ \\
Age (years) & $45.26 \pm 12.89$ & $50.77 \pm 14.69$ & 0.658 & 0.482 \\
BMI $\left(\mathrm{kg} / \mathrm{m}^{2}\right)$ & $29.98 \pm 4.38$ & $30.13 \pm 4.32$ & 0.075 & 0.768 \\
EST-exposed group $(\%)$ & $87(58.89)$ & $19(30.56)$ & 6.728 & $0.009^{*}$ \\
Diabetes & 12 & 5 & 0.967 & 0.355 \\
Hypertension & 25 & 31 & 3.005 & 0.079 \\
Cardiovascular & 21 & 13 & 0.074 & 0.783 \\
\hline
\end{tabular}

${ }^{*} p<0.05,{ }^{* *} p<0.01$

\section{Contrastive analysis of somnolence and PSG of OSAHS within the EST-exposed and non-exposed groups}

Compared with non-exposed OSAHS group, OSAHS group exposed to EST had more somnolence, higher AHI, lower oxygen saturation, and longer apnea pause time and hypopnea time ( $p<0.05$; Table 5). The exposed group scored higher on the ESS than the non-exposed group, which said there was more drowsiness. The reported SD values are highly overlapping in case longest apnea time in Table 5, but statistical processing was reliable.

Table 5. Comparison of ESS scores and PSG between OSAHS group exposed to EST and unexposed OSAHS group

\begin{tabular}{c|c|c|c|c}
\hline Parameter & $\begin{array}{c}\text { OSAHS group } \\
\text { exposed to EST }\end{array}$ & $\begin{array}{c}\text { OSAHS group } \\
\text { unexposed to ETS }\end{array}$ & $\begin{array}{c}\text { Test } \\
\text { value }\end{array}$ & $\boldsymbol{p}$ \\
\hline Number & 87 & 61 & & \\
ESS score & $\mathbf{9 . 6 5} \pm \mathbf{5 . 1 9}$ & $\mathbf{7 . 4 3} \pm \mathbf{4 . 7 9}$ & $\mathbf{2 . 3 5 9}$ & $\mathbf{0 . 0 2 2}^{*}$ \\
$\mathrm{AHI}(\mathrm{time} / \mathrm{h})$ & $\mathbf{4 6 . 3 3} \pm \mathbf{2 1 . 0 3}$ & $\mathbf{3 5 . 7 8} \pm \mathbf{2 6 . 3 7}$ & $\mathbf{2 . 1 6 9}$ & $\mathbf{0 . 0 4 3}^{*}$ \\
$\mathrm{LSaO}_{2}(\%)$ & $\mathbf{7 2 . 8 8} \pm \mathbf{3 . 6 4}$ & $\mathbf{7 8 . 9 2} \pm \mathbf{8 . 4 8}$ & $\mathbf{2 2 . 2 8}$ & $\mathbf{0 . 0 0 1}^{* *}$ \\
$\mathrm{MSaO}_{2}(\%)$ & $\mathbf{9 0 . 1 8} \pm \mathbf{4 . 3 2}$ & $\mathbf{9 2 . 2 5} \pm \mathbf{2 . 7 7}$ & $\mathbf{- 2 . 4 1 7}$ & $\mathbf{0 . 0 1 7}^{*}$ \\
Longest apnea time (s) & $\mathbf{7 7 . 6 5} \pm \mathbf{3 7 . 3 5}$ & $\mathbf{6 1 . 0 2} \pm \mathbf{3 3 . 4 2}$ & $\mathbf{2 . 1 3 8}$ & $\mathbf{0 . 0 4 6}^{*}$ \\
Longest hypoventilation time (s) & $\mathbf{9 9 . 2 7} \pm \mathbf{4 3 . 2 1}$ & $\mathbf{7 8 . 8 8} \pm \mathbf{4 1 . 5 0}$ & $\mathbf{- 2 . 2 5 0}$ & $\mathbf{0 . 0 4 0}^{*}$ \\
\hline${ }^{*} p<0.05,{ }^{* *} p<0.01$ &
\end{tabular}

\section{Comparison of characteristics, ESS scores and PSG in OSAHS with different exposure levels}

The groups were divided into ETS-exposed group and the non-ETS-exposed group, and the ETS-exposed subjects were classified into mid-ETS (tobacco smoke in the house but not in the same room as patient) and heavy-ETS (tobacco smoke in the same room as patient). In the ETS-exposed group, 25 OSAHS subjects with mid-ETS exposure, and 62 OSAHS subjects with heavy-ETS exposure were included. There were no significant differences in age and BMI between the two groups. Compared with OSAHS patients with mid-ETS exposure, OSAHS patients with heavy-ETS exposure 
had more ESS scores, greater AHI, lower oxygen saturation and longer apnea pause time $(p<0.05)$. There were no significant differences in longest hypopnea times $(p>0.05)$ between the two groups, as shown in Table 6.

Table 6. Comparison of ESS scores and PSG between OSAHS group with mid-ETS exposure and OSAHS group with heavy-ETS exposure

\begin{tabular}{c|c|c|c|c}
\hline Parameter & $\begin{array}{c}\text { OSAHS group with } \\
\text { mid-ETS exposure }\end{array}$ & $\begin{array}{c}\text { OSAHS group with } \\
\text { heavy-ETS exposure }\end{array}$ & $\begin{array}{c}\text { Test } \\
\text { value }\end{array}$ & $\boldsymbol{p}$ \\
\hline Number & 25 & 62 & & \\
$\mathrm{ESS}$ score & $\mathbf{8 . 5 7} \pm \mathbf{5 . 8 8}$ & $\mathbf{9 . 6 6} \pm \mathbf{6 . 0 7}$ & $\mathbf{2 . 5 0 5}$ & $\mathbf{0 . 0 1 2}^{*}$ \\
$\mathrm{AHI}(\mathrm{time} / \mathrm{h})$ & $\mathbf{3 8 . 3 9} \pm \mathbf{2 2 . 1 5}$ & $\mathbf{5 5 . 8 9} \pm \mathbf{2 6 1 7}$ & $\mathbf{- 2 . 4 4 7}$ & $\mathbf{0 . 0 2 2}^{*}$ \\
$\mathrm{LSaO}_{2}(\%)$ & $\mathbf{7 8 . 0 7} \pm \mathbf{1 2 . 8 3}$ & $\mathbf{6 8 . 8 7} \pm \mathbf{1 4 . 5 8}$ & $\mathbf{2 . 2 8 9}$ & $\mathbf{0 . 0 2 5}^{*}$ \\
$\mathrm{MSaO}_{2}(\%)$ & $\mathbf{8 9 . 9 7} \pm \mathbf{4 . 2 6}$ & $\mathbf{9 2 . 1 9} \pm \mathbf{2 . 7 3}$ & $\mathbf{- 2 . 6 8 2}$ & $\mathbf{0 . 0 1 1}^{*}$ \\
Longest apnea time (s) & $\mathbf{6 3 . 5 7} \pm \mathbf{3 2 . 4 7}$ & $\mathbf{9 3 . 9 6} \pm \mathbf{3 7 . 5 5}$ & $\mathbf{- 2 . 7 9 3}$ & $\mathbf{0 . 0 3 8}^{*}$ \\
Longest hypoventilation time (s) & $\mathbf{7 8 . 1 5} \pm \mathbf{4 2 . 2 6}$ & $\mathbf{9 7 . 8 7} \pm \mathbf{4 4 . 5 7}$ & $\mathbf{- 2 . 0 0 1}$ & $\mathbf{0 . 0 7 3}$ \\
\hline${ }^{*} p<0.05,{ }^{* *} p<0.01$ & \multicolumn{4}{l}{}
\end{tabular}

\section{Discussion}

Obstructive sleep apnea hypopnea syndrome (OSAHS) is a common sleep-disorder in the general population. It is one of the causes of drug resistance, refractory hypertension, and heart failure. If it is not treated effectively for a long time, it results in irreparable damage to multiple organs. The disorder seriously affects the quality of life and longevity of patients. The present study compared the ESS scores and AHI index of OSAHS patients exposed to ETS with those of OSAHS patients who were not exposed to ETS. In addition, the ESS score and PSG data of the OSAHS subjects exposed to mid-ETS were compared with those of OSAHS patients exposed to heavy-ETS. The study was aimed at providing scientific data and basis for adjunct clinical intervention in OSAHS. Long-term exposure to EST reduces the sensitivity of tissues to hypoxia and degrades the capacity to recover from conditions caused by hypoxia. These pathological changes impair the ability of the body to recover automatically from apnea when it occurs (Coggins, 2008; Owili et al., 2018; Van et al., 2018). This will result in a longer apnea and a long-term decline in blood oxygen saturation associated with it. It has been reported that patients with OSAHS exposed to mid-EST experienced longer and lower nocturnal hypoxemia than non-exposed OSAHS patients (Berman et al., 2018). In the treatment of OSAHS patients, doctors should, through joint interventions in multiple disciplines, pay attention to the patient's environmental status, and actively persuade them to improve the environment so as to reduce the occurrence of long-term complications of the disorder (Nachamkin, 2012; Li et al., 2018; Maskarinec et al., 2000). Improving the home environment can be used as an adjunct to conventional therapy for OSAHS.

The sample size used in this study was small. Moreover, retrospective studies may have recall bias. To obtain more reliable data, it is important to use a larger sample size and more representative study locations (Yang et al., 2018; Nurminen and Jaakkola, 2004). It is also important to apply more stringent control conditions in the study of EST and improvement of OSAHS. The relationship between ETS and OSAHS is expected to be further elucidated through animal experiments. 


\section{Conclusion}

This study shows that there is a certain correlation between EST and OSAHS. Compared with subjects who were not exposed to ETS, patients exposed to EST are more susceptible to OSAHS, and the longer the exposure time, the more severe the condition. This study found that the BMI of the OSAHS group was significantly higher than that of the non-OSAHS group, which is consistent with the currently wellrecognized high incidence of obesity and OSAHS. The study found that the ETS exposed group had higher ESS, higher AHI, lower oxygen saturation, and longer apnea time and hypopnea time. Compared with non-smokers, the current smoking population has longer sleep latency, shorter sleep time, longer I-stage sleep time, and slower slowwave sleep time. The sleep characteristics of OSAHS patients are prolonged latency, sleep fragmentation, and prolonged stage I sleep. III stage IV sleep reduction. To a certain extent, this also explains the impact of ETS exposure on sleep.

Thus, OSAHS may be mitigated by improving the environment. Families are the main places where tobacco smoke is exposed. Public health policies need to focus on family smoking ban. To publicize and pay attention to the harm of EST, we should actively advocate smoking cessation, strengthen the publicity of the hazards of passive smoking, control the impact of EST on OSAHS, and have great significance in preventing OSAHS.

\section{REFERENCES}

[1] Aviado, D. M. (1996): Cardiovascular disease and occupational exposure to environmental tobacco smoke. - American Industrial Hygiene Association Journal 57(3): 285-294.

[2] Barnes, R. L., Hammond, S. K., Glantz, S. A. (2006): The tobacco industry's role in the 16 cities study of secondhand tobacco smoke: do the data support the stated conclusions? - Environmental Health Perspectives 114(12): 1890-1897.

[3] Berman, T., Barnettitzhaki, Z., Mery, N., Keinanboker, L., Shimony, T., Goldsmith, R., Göen, T., Geva, H., Rosen, L. (2018): Exposure to environmental tobacco smoke in nonsmoking adults in Israel: results of the second Israel biomonitoring survey. - Israel Journal of Health Policy Research 7(1): 33.

[4] Coggins, C. R. E. (2008): The Osha review of Animal inhalation studies with environmental tobacco smoke. - Inhalation Toxicology 8(8): 819-830.

[5] Davidson, M., Reed, S. (2018): Preliminary assessment of real-time air quality instruments for monitoring environmental tobacco smoke. - British Actuarial Journal 3(2): 547-614.

[6] Elliott, L., Arbes, S. J., Harvey, E. S., Lee, R. C., Salo, P. M., Cohn, R. D., London, S. J., Zeldin, D. C. (2007): Dust weight and asthma prevalence in the National Survey of Lead and Allergens in Housing (NSLAH). - Environmental Health Perspectives 115(2): 215220.

[7] Jain, R. B. (2018): Rates of exposure to environmental tobacco smoke from various indoor environments among US children and nonsmoker adolescents and adults. Environmental Science \& Pollution Research 25(4): 17002-17011.

[8] Jenkins, R. A., Counts, R. W. (1999): Occupational exposure to environmental tobacco smoke: results of two personal exposure studies. - Environmental Health Perspectives 107(2): 341-348.

[9] Li, M., Liu, X., Zhang, L. (2018): The relationship of indoor coal use and environmental tobacco smoke exposure with lung cancer in China: A meta-analysis. - Journal of Cancer Research \& Therapeutics 14: 7-13. 
[10] Maskarinec, M. P., Jenkins, R. A., Counts, R. W., Dindal, A. B. (2000): Determination of exposure to environmental tobacco smoke in restaurant and tavern workers in one US city. - Journal of Exposure Analysis \& Environmental Epidemiology 10(1): 36.

[11] Muggli, M. E., Hurt, R. D., Repace, J. (2004): The tobacco industry's political efforts to derail the EPA report on ETS. - American Journal of Preventive Medicine 26(2): 167177.

[12] Nachamkin, E. W. (2012): An examination of secondhand smoke in a sample of Atlanta hospitality venues and their compliance with the Georgia Smokefree Air Act. - Journal of Animal Science 84(2): 291-299.

[13] Nurminen, M. M., Jaakkola, M. S. (2004): On the estimation of lung cancer mortality caused by occupational exposure to environmental tobacco smoke in Finland. - Journal of Occupational \& Environmental Medicine 46(2): 93-95.

[14] Owili, P., Muga, M., Kuo, H. W. (2018): Gender difference in the association between environmental tobacco smoke and birth weight in Africa. - International Journal of Environmental Research \& Public Health 15(7): 1409.

[15] Rando, R., Poovey, H., Gibson, R., Lehrer, S. (1999): 136. Laboratory and field study of emission of environmental tobacco smoke from smokeless cigarette. - American Industrial Hygiene Conference and Exposition, Toronto, Ontario, June 5-11.

[16] Steenland, K. (1999): Risk assessment for heart disease and workplace ETS exposure among nonsmokers. - Environmental Health Perspectives 107(6): 859-863.

[17] Theweny, T. S. (2015): Measurement of thirdhand smoke in a casino: surface nicotine wipes pre and post smoking ban. - Thesis in partial fulfilment of the requirements for the degree Master of Social Work and Master of Public Health. San Diego State University, San Diego, CA.

[18] Thun, M., Henley, J., Apicella, L. (1999): Epidemiologic studies of fatal and nonfatal cardiovascular disease and ETS exposure from spousal smoking. - Environmental Health Perspectives 107(6): 841-846.

[19] Van, F. O., Jochems, S., Wesselius, A., Van, F. S., Bryan, R. T., Zeegers, M. P. (2018): A stratified meta-analysis of the association between exposure to environmental tobacco smoke during childhood and adulthood and urothelial bladder cancer risk. - International Journal of Environmental Research \& Public Health 15(4): 569.

[20] Yang, H. S., Lim, H., Choi, J., Bae, S., Kim, Y., Kwon, H. J., Ha, M. (2018): Environmental tobacco smoke exposure at home and attributable problem behaviors in Korean children and adolescents for 2012-2014 in a nationally representative survey. Journal of Korean Medical Science 33(36): 229. 